"Ion effects in hydrogen-induced blistering of Mo/Si multilayers"

A. S. Kuznetsov, M. A. Gleeson and F. Bijkerk

Journal of Applied Physics 114(11) (2013) 113507-113512.

http://dx.doi.org/10.1063/1.4821844

\title{
Ion effects in hydrogen-induced blistering of Mo/Si multilayers
}

\author{
A S Kuznetsov ${ }^{1}$, M A Gleeson ${ }^{1,}$ a , and F Bijkerk ${ }^{1,2}$ \\ ${ }^{1}$ FOM Institute DIFFER - Dutch Institute for Fundamental Energy Research, Postbus 1207, 3430 BE \\ Nieuwegein, The Netherlands. (www.differ.nl) \\ ${ }^{2}$ MESA+ Institute for Nanotechnology, University of Twente, Postbus 217, 7500 AE Enschede, The \\ Netherlands.
}

\begin{abstract}
The role that energetic ( $>800 \mathrm{eV}$ ) hydrogen ions play in inducing and modifying the formation of blisters in nanoscale Mo/Si multilayer samples is investigated. Such samples are confirmed to be susceptible to blistering by two separate mechanisms. The first is attributed to the segregation of $\mathrm{H}$ atoms to voids and vacancies associated with the outermost Mo layer, driving blister formation in the form of $\mathrm{H}_{2}$ filled bubbles. This process can occur in the absence of ions. A second blister distribution emerges when energetic ions are present in the irradiating flux. This is attributed to an ion-induced vacancy clustering mechanism that produces void blisters. The defects and strained states associated with the Mo-on-Si interfaces provide the preferred nucleation points for blistering in both cases. The effects of ions are ascribed to promotion of hydrogen uptake and mobility, in particular through the Si layers; to the generation of additional mobile species in the Si and Mo layers; and to the creation of new blister nucleation points. In addition to directly stimulating blistering via vacancy clustering, ions modify the development of $\mathrm{H}_{2}$-filled blisters.

This is most evident in the formation of multi-component structures due to overlapping delaminations at different layer interfaces. This affect is attributed to the introduction of active transport of hydrogen from the $\mathrm{H}_{2}$ filled blisters across the outermost Mo-on-Si interface to the underlying layers. Ion-induced variations in hydrogen uptake and distribution and in the rates of blister nucleation and growth produce lateral differences in blister size and areal number density that create a macroscopic concentric pattern across the surface.
\end{abstract}

\footnotetext{
a Author to whom correspondence should be addressed. Electronic mail: m.a.gleeson@differ.nl
} 


\section{Introduction}

The interaction of hydrogen with materials is one of the most basic, yet challenging, of research topics. While much is understood, many open and active questions remain regarding the manner in which hydrogen behaves within materials. One possible consequence of the interaction is the formation of blisters. Depending on the material type and composition, exposure conditions ranging from energetic ion implantation to (plasma-)hydrogenation, or combinations thereof, can induce blistering [1-3]. Understanding the underlying phenomena is relevant for such diverse applications as the development of plasma-facing materials for fusion reactors [4-8], thin film deposition [9-11], and silicon-on-insulator layer transfer processes in the microelectronics industry [2, 12-14].

Deposited layers can exhibit a susceptibility to blistering that is dependent on the layer structure and composition, as well as on the presence of interfaces. For instance, localized delamination can be achieved with buried strained layers [14-16]. Multilayer (ML) structures are an extreme example of a deposited layer system, with multiple interfaces at various depths available as potential blister nucleation sites. The formation of blisters in such structures has potential consequences for several applications. Various authors have addressed He-induced blister formation in MLs amongst other consequences of exposure to harsh environments (see e.g. [17-20]). With regard to effects induced by hydrogen, surface damage in the form of blisters and craters (collapsed blisters) has been observed upon annealing of hydrogenated Si/Ge ML structures [21, 22], while in-plane fracturing and layer delamination has been observed during cycling of $\mathrm{Mg} / \mathrm{FeTi}$ hydrogen storage MLs [23]. Blistering occurred during hydrogen ion bombardment of ML mirrors intended for solar research [24] and during hydrogenation of Co/Pd MLs studied in the context of data storage [25].

In a previous paper [26] we reported on how the extent and type of hydrogen-induced blistering of Mo/Si ML samples was dependent on the composition of the incident flux. The individual layers of these samples are comprised of amorphous silicon ( $a$-Si) and polycrystalline Mo, with intermixed interface regions (see Discussion). A great deal of research spanning many decades has focused on the interaction of hydrogen with these materials on an individual basis. Silicon is primarily studied in relation to the micro-electronics/semi-conductor industry and the development of solar cells technology [27, 28], while molybdenum is studied in the context of 


\section{"Ion effects in hydrogen-induced blistering of Mo/Si multilayers" \\ A. S. Kuznetsov, M. A. Gleeson and F. Bijkerk \\ Journal of Applied Physics 114(11) (2013) 113507-113512. \\ http://dx.doi.org/10.1063/1.4821844}

fusion and plasma-facing wall materials [5, 29] or as part of broader investigations of hydrogen with a range of metals [30,31]. An overview of some studies on hydrogen interactions with semiconductors and metals that are of relevance to the current work is provided in the following paragraphs.

The configurations of hydrogen atoms in an $a$-Si network span a wide range of energies [3234], with the bulk of them acting to passivate dangling bonds. Due to the bond strengths involved, direct diffusion of such species is unlikely [35]. Su and Pantelides [36] suggest a floating bond-H complex as the "migrating species" while Abtew et al. [37] illustrated a "fluctuating bond center detachment" mechanism for the diffusion of hydrogen bonded within a Si network. Both mechanisms require an active site in the form of an under-coordinated Si atom for transport of $\mathrm{H}$ through the layer. Thus, motion of $\mathrm{H}$ in $a-S i$ is determined by availability of the necessary defect structures and ultimately involves only a minority of the total hydrogen in the system.

$A b$ initio simulations of $\mathrm{H}$ in $a-S i$ and hydrogenated amorphous silicon reveal a strong tendency to attacked strained bond structures in the network [28]. The effect of ions on $a$-Si can be understood in terms of their ability to introduce and remove the defect structures necessary for efficient transport of hydrogen through the network. Sadot et al. [38] reported stress relaxations of deposited SiGe layers during $\mathrm{H}^{+}$implantation as a result of bond breaking. Ion irradiation stimulated the release of hydrogen implanted in Si from strongly-bonded configurations resulting in diffusion and attachment to vacancy defects [39]. Thus it enhanced the effective mobility of H. Subsequent annealing resulted in blister formation that did not occur in the case of unirradiated samples.

The interaction of hydrogen with metals is typically dominated by the material structure. For example, the solubility and diffusion of hydrogen in amorphous and nano-crystalline metals differ significantly from their crystalline and polycrystalline counterparts [40-43]. The diffusion coefficient typically has a strong dependence on the hydrogen concentration, being less than that of the crystalline form at low concentrations, but increasing and exceeding it at higher concentrations [43].

Rigato et al. [44] reported negligible $\mathrm{H}$ retention in thick Mo layers deposited in a $\mathrm{H}_{2}$ containing atmosphere. This was attributed to the high positive heat of solution of $\mathrm{H}$ in bulk Mo 


\section{"Ion effects in hydrogen-induced blistering of Mo/Si multilayers" \\ A. S. Kuznetsov, M. A. Gleeson and F. Bijkerk \\ Journal of Applied Physics 114(11) (2013) 113507-113512. \\ http://dx.doi.org/10.1063/1.4821844}

[30]. The only consequence of depositing Mo layers in a hydrogen-atmosphere that they reported was a slight reduction in the integrated X-ray diffraction peak intensity of the (110) Mo reflection. Trapping of hydrogen at ion-induced defects in Mo is commonly observed [45, 46]. Nagata and co-workers [47, 48] found substantial localized hydrogen uptake (10-20 at.\%) in Heimplanted Mo single crystal samples. The trapped $\mathrm{H}$ was associated with the nanostructure around implanted He atoms. In a similar vein, Hoshihira et al. reported tritium accumulation near grain boundaries of polycrystalline Mo samples after exposure to an AC glow discharge [29]. They identified two hydrogen blistering mechanisms. One involved exfoliation as a result of $\mathrm{H}$ accumulation at grain boundaries or clustered defects, while the other was the result of bubble coalescence.

In general the most stable form of metal-H alloys is a defect structure containing a large number of vacancies [49]. Calculations on the configuration and binding energies of $\mathrm{H}$ atoms in transition metal mono-vacancies show that up to 12 atoms can be accommodated in Mo [31]. Incorporation of additional atoms resulted in $\mathrm{H}_{2}$ formation. Based on first principles calculation, Zhou et al [50] reported an increased H solubility in anisotropic strained BCC metals (W, Mo, $\mathrm{Fe}, \mathrm{Cr}$ ) over a wide range of both compressive and tensile values. Only over a small region of compressive strain was the solubility slightly less than that of the strain free metal. Hence, local anisotropic strain fields had the potential to drive the segregation of hydrogen to the vicinity of existing bubbles almost independent of the sign of the strain. They proposed that the resultant strain increase could stimulate further segregation resulting in a cascaded effect of bubble growth.

The preceding paragraphs give an indication of the wealth of existing information relating to the materials present in our samples. However, it is important to bear in mind that we are dealing with materials that are constrained by the nanometer scale thickness of the individual layers. The constraining of materials within thin-layered structures can have a dramatic impact on the interaction with hydrogen [51, 52]. The behavior of such systems cannot necessarily be inferred from bulk material properties. The H-related properties of the ML will be determined by the local strain states in the individual layers, by the presence and density of defect sites and (self)interstitials, by the crystallinity (and crystal size) of the layers, and by the degree of intermixing between individual layers. These parameters will be dependent on the precise details of the layer 
composition and on the deposition conditions (e.g. residual gas composition [53]) and procedures. Thus, the interactions of hydrogen with nano-scale MLs involve numerous inherent complexities, in addition to those of the single-material systems.

The current work expands on earlier reports $[26,54,55]$ by focusing on the specific roles that energetic ions play in inducing and modifying blistering of Mo/Si MLs. These ions are an incidental consequence of the mode of operation of the particle source and have energies in the 800-1000 eV range. We have not determined a lower threshold for the influences of energetic particles that are addressed in this work. Similar effects have been observed at $650 \mathrm{eV}$ (higher fluence) [26]. The lower energy threshold will be related to the characteristic penetration depths and the thickness of the layers under irradiation. From an application perspective, the energies present in the current exposures are relevant for layer processing technologies such as Siliconon-Insulator [56]. The original "Smart-Cut" technique utilizes keV ion energies [57], but this becomes increasingly problematic as transferred layer thickness requirements become more demanding. More recent developments involve the use of buried strained layers and plasma hydrogenation with biasing in the range of -100 to $-500 \mathrm{~V}$ [14-16]. Incidental energetic ions irradiation of MLs can also occur in the intended operating environment. $1 \mathrm{keV}$ hydrogen irradiation of Mo/Si MLs has been used to simulate the effects of the quite solar wind plasma on optical components of solar research instrumentation [24], while simulation of near-surface lightinduced plasma production showed maximum ion-impact energies of $100 \mathrm{eV}$ [58].

We elucidate the influences of ions by studying the development of blisters as a function of exposure time and by comparing the effects of exposures performed with and without ions in the irradiating flux. The presence of two separate blistering processes is confirmed. These are assigned to $\mathrm{H}_{2}$ bubble formation, which does not require energetic ions, and to a vacancy clustering mechanism, which does. The factors that drive the localization of blisters and ioninduced modification of blister development on both a microscopic and a macroscopic scale are discussed.

\section{Experimental details}

The MLs under investigation were composed of alternating layers of molybdenum and silicon. The stack was terminated by a silicon layer. The thickness of the individual layers was 


\section{"Ion effects in hydrogen-induced blistering of Mo/Si multilayers" \\ A. S. Kuznetsov, M. A. Gleeson and F. Bijkerk \\ Journal of Applied Physics 114(11) (2013) 113507-113512. \\ http://dx.doi.org/10.1063/1.4821844}

3 $\mathrm{nm}$ for Mo and 4 $\mathrm{nm}$ for Si. These were deposited by magnetron sputtering using in-house deposition facilities. An additional ion polishing step was applied to the Si layers in order to minimize the development of interfacial roughness during the course of the deposition [59, 60]. All samples were exposed to atmosphere after deposition. Consequently, a native oxide layer is formed at the top of the outermost Si layer.

No special cleaning was applied to the samples prior to hydrogen irradiation. Samples were irradiated with the hydrogen flux generated by a capillary-type thermal gas cracker (TC-50, Oxford Applied Research [61]). The cracker was mounted in an exposure chamber that was operated at a base pressure of $\sim 1 \times 10^{-8}$ mbar. In the current study, the source power was kept constant (55 W) and a $\mathrm{H}_{2}$ flow of 1 sccm was used. The background pressure in the chamber during exposures was $\sim 5 \times 10^{-5}$ mbar. Samples were exposed at elevated temperatures, typically ranging from $358 \mathrm{~K}$ to $373 \mathrm{~K}$. Details of the sample temperature during exposures are given at the relevant points in the Results section. Increasing the sample temperature typically resulted in accelerated manifestation of blisters.

The thermal cracker is nominally a source of only neutral species. However, during exposures a measurable drain current was collected by the sample. The total current was dependent on the operating condition: in the current case it was $~ 75 \mathrm{nA}$. The ion energies, as determined using a retarding field analyzer (RFA), were predominantly in the range from 800 to $1000 \mathrm{eV}$ (see Results). In addition to exposing samples to the unmodified flux from the source, exposures were also performed with the insertion of a deflector plate close to the sample and parallel to the irradiating beam direction. By applying a bias of $-1100 \mathrm{~V}$ to this plate, charged particles were deflected from the incident flux resulting in the net current on the sample dropping to a fraction of a nA. The influence of ions could thus be distinguished by comparing the effects of otherwise equivalent exposures performed with (“neutral-only”) and without (“ion+neutral”) the biased plate.

Damage to exposed samples was evident to the naked eye prior to breaking vacuum. Postexposure analysis required transport through air. Samples were analyzed by optical microscopy and atomic force microscopy (AFM) to identify and quantify structural changes to the surface. Resonant nuclear reaction analysis (NRA) utilizing the ${ }^{1} \mathrm{H}\left({ }^{15} \mathrm{~N}, \alpha \gamma\right){ }^{12} \mathrm{C}$ reaction was used to produce depth profiles of the H-content of selected samples. Gamma rays, arising from the 


\section{"Ion effects in hydrogen-induced blistering of Mo/Si multilayers" \\ A. S. Kuznetsov, M. A. Gleeson and F. Bijkerk \\ Journal of Applied Physics 114(11) (2013) 113507-113512. \\ http://dx.doi.org/10.1063/1.4821844}

resonance in the nuclear reaction at $6.385 \mathrm{MeV}$, were detected with a $4 \mathrm{x} 4$ inch bismuth germanate scintillation detector placed directly behind the sample. The correspondence between the incident NRA particle energy and the reaction depth was assigned on the basis of a layer profile derived from Rutherford Backscattering Spectrometry (RBS) measurements. These were performed with a $2 \mathrm{MeV}$ He beam at normal incidence to the sample surface. The spectra were registered at a scattering angle of $170^{\circ}$ with a Canberra passivated implanted planar silicon (PIPS) detector. The post-NRA hydrogen content of the samples was measured by elastic recoil detection (ERD) analysis using a 3.5 MV particle accelerator (Singletron type). The angle of incidence of the He beam was $15^{\circ}$ with respect to the sample surface. Recoiled hydrogen was detected by a Canberra PIPS detector at an angle of $25^{\circ}$ with respect to the incident beam direction. A $9 \mu \mathrm{m}$ thick Mylar foil was mounted in front of the detector to block scattered He particles. The depth resolution of the ERD analysis was $\sim 30 \mathrm{~nm}$.

\section{Results}

\section{Flux profiles}

Figure 1(a) shows an RFA ion energy profile acquired along the central axis of the source. The majority of ions have an energy in the range from $800-1000 \mathrm{eV}$. Two distinct peaks are evident: one centered at $\sim 860 \mathrm{eV}$ and one at $\sim 1000 \mathrm{eV}$. Heating of the thermal cracker is provided by an electron flux that is accelerated from a hot filament to a capillary tube that is biased at $+1000 \mathrm{~V}$ [61]. Thus, the energies measured are consistent with repulsion of ions created in the vicinity of the capillary. The origin of the two peaks is not obvious. However, comparison of lateral ion profiles (see below) suggests that the $860 \mathrm{eV}$ peak is due to ionization of hydrogen in the direct flow from the capillary while the $1000 \mathrm{eV}$ peak is due to ionization of background hydrogen. The former implies a mixture of atomic and molecular ions, while the latter would produce primarily molecular ions.

The ion flux across the sample surface was not uniform. Figure 1(b) shows RFA measurements of lateral ion profiles. The total ion profile $(0-1010 \mathrm{eV})$ has a maximum in the center of the beam and decreases towards the periphery. Individual profiles for the energy ranges $<800 \mathrm{eV}$, from $800-960 \mathrm{eV}$, and from $960-1010 \mathrm{eV}$ are also shown. The $<800 \mathrm{eV}$ profile is 


\section{"Ion effects in hydrogen-induced blistering of Mo/Si multilayers" \\ A. S. Kuznetsov, M. A. Gleeson and F. Bijkerk \\ Journal of Applied Physics 114(11) (2013) 113507-113512. \\ http://dx.doi.org/10.1063/1.4821844}

essentially flat; the 800-960 eV profile is strongly peaked along the beam axis; while the 9601010 eV profile has a broad peaked distributions.

\section{Blister formation during "ion+neutral" exposures.}

Direct exposure of samples to the unmodified hydrogen flux from the capillary cracker resulted in the development of distinctive circular damage patterns on the surface. The effect is illustrated in Figure 2, which shows photographs of $\sim 12 \times 12 \mathrm{~mm}^{2}$ samples taken after (a) 3 and (b) 5 hour exposures, respectively. The sample temperature during these exposures ranged from $298 \mathrm{~K}$ to $358 \mathrm{~K}$. This range was the result of direct heating from the thermal cracker. At the start of an exposure the sample was at room temperature. After $\sim 45$ minutes the temperature stabilized at $\sim 358 \mathrm{~K}$.

The images in Figure 2 correctly reproduce the patterns that were discernable by eye, although the photographic method exaggerates the contrast between different regions. The apparent color is also an artifact of the photographic method; to the naked eye the damaged areas appeared whitish. The area indicates as “deposition clamp” represents a region of bare Si wafer, which was exposed to the hydrogen flux, while "exposure clamp” is a region of deposited ML that was covered during the exposure.

For the purposes of the current work the damage patterns observed can be divided into three regions. On Figure 2 these are characterized as a "central spot", an "intermediate ring”, and a “periphery region”. On both samples the central spot had the appearance of being the most severely damaged. The periphery region exhibited a diffuse discoloration that was less intense than that of the central spot. The intermediate ring was the slowest to exhibit visible damage. After 3 hours this portion of the surface retained its original mirror-like reflectivity and to the eye appeared unaffected by the exposure.

Inspection of the samples using white-light interferometry and AFM revealed the presence of blister-like structures in all three regions of both samples. Thus, blisters were also present in the "intermediate ring” after the 3-hour exposure, although both their size and the areal number density were very small, indicating that the surface manifestation of blistering has only commenced.

The $25 \times 25 \mu \mathrm{m}^{2}$ AFM images that were reported in [26] were collected from the central spots of the two samples shown in Figure 2. Figure 3 shows $5 \times 5 \mu \mathrm{m}^{2}$ AFM images collected from the 


\section{"Ion effects in hydrogen-induced blistering of Mo/Si multilayers" \\ A. S. Kuznetsov, M. A. Gleeson and F. Bijkerk \\ Journal of Applied Physics 114(11) (2013) 113507-113512. \\ http://dx.doi.org/10.1063/1.4821844}

central spots ((a)\&(c)) and the periphery ((b)\&(d)) regions of these samples. After the 3-hour exposure (Figure 3(a)\&(b)) the blisters in the two regions appear similar, although those in the periphery are somewhat larger than those in the central spot. After the 5-hour exposure the blister density in the periphery is essentially unchanged but the individual blisters have increased in size (Figure 3(d)). In contrast, the density of blisters in the central region has increased dramatically due to the emergence of a large number of comparatively small blisters (Figure 3(c)). The central spot now contains fewer blisters that are of comparable size to those evident in the periphery region of the same sample. Thus, although both regions exhibit a continued growth of existing blisters when the exposure time is extended, the growth rates differ significantly.

Instances of “double-blister” structures are visible in Figure 3(c) (see arrows). Such structures arise from overlapping delaminations occurring at different interfaces [26]. They were only observed in the central spot after the 5 hour exposure. Transmission electron microscopy (TEM) imaging reveals that these blisters are the result of delamination occurring at the two outermost Mo-on-Si interfaces (see [26, 54]). Thus far no instance of delamination at a Si-on-Mo interface or at deeper Si-on-Mo interfaces has been observed.

The blisters seen in Figure 3(a),(b)\&(d) are consistent with a single size distribution. Two distributions were evident only in the central spot after the 5 hour exposure. As outlined in [26], the two distributions could be distinguished on the basis of their relative size and their aspect ratio (e.g. radius to Z-height), with the larger of the two being attributed primarily to the uptake of neutral hydrogen and the smaller to direct ion-induced processes. Throughout this manuscript, these two distributions will be referred to by the shorthand labels "thermal neutral" and "ioninduced”, respectively. The validity of these labels will be considered in light of the results presented.

Figure 4 shows plots of individual blister volume versus area covered. This data is derived from $25 \times 25 \mu \mathrm{m}^{2}$ AFM scans collected from the samples shown in Figure 2. Figure 4(a) shows data from the central spot and Figure 4(b) from the periphery region. The solid line is the result of fitting the 5 hour exposure data points on Figure 4(b) with an equation of the form: $y=a \times\left(x^{b}\right)$. This trace is reproduced on both panels as a reference curve to aid comparison between the datasets. The data from the periphery region after the 3 hour exposure also exhibits a good correlation with this curve. In this region the effect of increasing the exposure time is simply 


\section{"Ion effects in hydrogen-induced blistering of Mo/Si multilayers" \\ A. S. Kuznetsov, M. A. Gleeson and F. Bijkerk \\ Journal of Applied Physics 114(11) (2013) 113507-113512. \\ http://dx.doi.org/10.1063/1.4821844}

additional blister growth, and all blisters are classified as "thermal neutral". The blisters in the central spot after the 3 hour exposure are qualitatively similar to those in the periphery region, although they are generally smaller and are "flatter" (as indicated by their position relative to the reference curve) than those in the periphery. These are also classified as "thermal neutral”.

Divergence of the blister development in the central spot after the 5 hour exposure is clearly evident from Figure 4(a). While some of the "thermal neutral" blisters in this region exhibit growth, the majority remain clustered at sizes comparable to those that are already present after 3 hours. There is increased scatter in these data points, but they remain consistently below the reference curve. Blisters classified as “ion-induced” have now also emerged (individual areas $<2 \times 10^{5} \mathrm{~nm}^{2}$; see insets). These generally lie above the reference curve, indicating that they are "taller" than the "thermal neutral” blisters in aspect ratio terms. It is also evident from the insets that a significant number of additional "thermal neutral” blisters have developed in the central spot after 5 hours. Similar effects are not apparent in the periphery region.

\section{“Ion+neutral” versus “neutral-only” exposures.}

To test the assignment of the "thermal neutral” (to neutral species) and "ion-induced" (to energetic ions) blister distributions, exposures were performed without and with utilization of the biased deflector plate ("ion+neutral" and "neutral-only", respectively). Note that for these exposures the cracker-sample distance was increased by $\sim 50 \mathrm{~mm}$. This reduced direct heating of the sample by the source. Instead, active heating was applied via a rear-mounted filament and the sample temperature was stabilized at $373 \mathrm{~K}$ before the start of the exposures. The most immediately obvious result of utilizing the biased plate was the absence of the circular damage pattern. After neutral-only exposures the exposed surface appeared uniformly damaged, whereas ion+neutral exposures still produced a concentric pattern.

Figure 5 shows $25 \times 25 \mu \mathrm{m}^{2}$ AFM scans acquired from the central spot position of samples after exposure to (a) the ion+neutral and (b) the neutral-only fluxes. The smaller of the two blister distributions is present after the ion+neutral exposure but is absent after the neutral-only exposure. Hence, the "ion-induced" nature of these features is confirmed. Furthermore, in the neutral-only case the ("thermal neutral”) blisters that do develop exhibit only a single-component structure, whereas after the ion+neutral exposure all "thermal neutral" blisters have a double- or multi-component structure. Thus, the development of such structures is also due to the presence 
of energetic ions in the irradiating flux. A single distribution of single-component blisters is observed in the periphery regions of both samples.

Figure 6 shows plots of individual blister volume versus area covered derived from 50×50 $\mu \mathrm{m}^{2}$ AFM scans from the "ion+neutral” and "neutral-only" exposed samples. Figure 6(a) compares the data from the central spot position of the two samples. The "ion-induced" blisters, are represented by a high density of points with area $<8 \times 10^{5} \mathrm{~nm}^{2}$. These are much larger than the corresponding blisters in figure 4(a). There are very few features in this size range in this region of the sample exposed to the neutral-only flux.

The reference curve from Figure 4(b) is again reproduced in these panels. Similar to Figure 4(a), the "ion-induced" blisters tend to lie above the reference curve. "Thermal neutral” blisters tend to lie below the reference curve after the ion+neutral exposure, while they have a reasonably good correspondence with it after the neutral-only exposure. The development of multicomponent "thermal neutral" blisters in the central spot when ions are present versus singlecomponent blisters when they are absent is again apparent from the relative scatter in these data points. The presence of ions leads to a general increase in both the volume of and the area covered by these blisters relative to when ions are absent.

In the periphery region both datasets have a reasonably good correspondence with the reference curve. However, the blisters are smaller when ions are present relative to when they are absent. In the case of the sample exposed to the neutral-only flux the blisters distributions in the periphery and central spot regions are very similar. In contrast, on the sample exposed to the ion+neutral flux the blisters in the periphery region are significantly smaller than the "thermal neutral" blisters in the central spot. This is in marked contrast to Figure 4, where the "thermal neutral" blisters are larger in the periphery region.

Figure 7 shows a comparison of (a) the blister court, (b) the total blister volume, and (c) the average blister volume for the three 5 hour exposed samples. The "ion-induced" and "thermal neutral" distributions are analyzed separately in the case of the two ion+neutral exposures. The essentially uniform blister development in the case of the neutral-only exposure is confirmed. The ion+neutral exposures produce large variations in blister number and volume across the sample surface. The much higher density of smaller "ion-induced" blisters produced in the 


\section{"Ion effects in hydrogen-induced blistering of Mo/Si multilayers" \\ A. S. Kuznetsov, M. A. Gleeson and F. Bijkerk \\ Journal of Applied Physics 114(11) (2013) 113507-113512. \\ http://dx.doi.org/10.1063/1.4821844}

central spot of the "original" 5 hour ion+neutral exposure (Figure 2(b)) is primarily due to the differences in sample temperature during exposure.

\section{H-content and depth profile}

Figure 8 shows a comparison of resonant NRA H-content profiles acquired from the central spot position of the "ion+neutral" and "neutral-only" exposed samples shown in Figure 5. The oscillatory profile with enhanced H-content in the Mo layers relative to the adjacent Si layers was seen previously [54]. The H-content in the outermost Si and Mo layers is elevated in the case of the neutral-only exposure relative to the ion+neutral exposure. However, post-NRA determination of the total $\mathrm{H}$-content in these samples by ERD yielded $\mathrm{H}$ concentrations of $\sim 1.4 \times 10^{16}$ at $/ \mathrm{cm}^{2}$ and $\sim 1.13 \times 10^{16}$ at $/ \mathrm{cm}^{2}$ in the outermost $\sim 30 \mathrm{~nm}$ of the samples exposed to the ion+neutral and the neutral-only fluxes, respectively. Thus, ERD indicates that the H-uptake is increased by $24 \%$ when ions are present, whereas the NRA data showed an enhancement of the local content in the outermost layers when they are absent. There was no indication of instability in the H-content of either sample during the course of the ERD measurements. A possible reason for the apparent discrepancy is that ions are promoting not only hydrogen uptake, but also enhancing its distribution and localization within the ML structure. Enhanced localization would be consistent with the increased blistering that is observed when ions are present. This should not affect the total content derived from ERD but, depending on the degree of localization as a function of depth, could potentially lead to an under-estimation in the case of the point-wise resonant NRA measurements. In particular, gaseous $\mathrm{H}_{2}$ trapped in void and blisters might be under-estimated.

\section{Discussion}

\section{The multilayer structure}

Structural and compositional details typical of Mo/Si MLs have been extensively investigated [59, 62-64]. The Si layers are amorphous, while the Mo layers are polycrystalline. The Mo crystallite size is determined by the layer thickness [64], hence these layers can be characterized as highly defective polycrystalline or nano-crystalline. Given the layer thicknesses involved, the material properties tend to be dominated by interfacial effects. An intermixed zone is formed at both interfaces during deposition. The interfacial zones are asymmetric with a thicker layer 


\section{"Ion effects in hydrogen-induced blistering of Mo/Si multilayers" \\ A. S. Kuznetsov, M. A. Gleeson and F. Bijkerk \\ Journal of Applied Physics 114(11) (2013) 113507-113512. \\ http://dx.doi.org/10.1063/1.4821844}

forming at Mo-on-Si interfaces than at Si-on-Mo interfaces [59, 65, 66]. This effect is primarily related to the crystallinity of the Mo layers. In the case of the Si-on-Mo interface, Si is deposited on polycrystalline Mo. This hinders intermixing and results in a rapid transition to the pure $a$-Si phase. More effective intermixing during Mo-on-Si deposition delays Mo recrystallization, resulting in a thicker interfacial zone [66, 67].

The deposited layers typically have significant internal stresses that arise due to the lattice mismatches at the interfaces. Since the layers are very thin, the individual materials do not relax back to their bulk properties before a new interface is initiated. The Si layers produce compressive lateral stress (i.e. they are tensilely-strained in-plane) while the Mo layers produce tensile lateral stress (compressively-strained in-plane) [68]. These macroscopic properties are determined by localized effects on the atomic scale, particularly those occurring in the interfacial regions where the effects of lattice mismatches are at their most extreme [64].

Hydrogen incorporation during deposition was reported to produce more compressive Si layers and more tensile Mo layers, with the effect on Mo layers being 2.5 times that on Si layers [53]. Similar effects produced by post-deposition incorporation of hydrogen, coupled with preferential accumulation in Mo, would introduce a strong driving force for Mo deformation in order to relax the overall strain on the layer.

Hydrogen distribution within individual layers will be dominated by the local chemical and structural composition and the associated lattice strains. Interfaces can play a significant role in the context of hydrogen retention. Thus, buried strained layers can be used to control the distribution and nucleation of hydrogen in order to produce a depth-localized layer detachment [14-16]. TEM imaging indicates that macroscopic blistering in the case of our neutral-only exposures is exclusive to the outermost Mo-on-Si interface. The comparatively high H-content in the outermost Mo layer suggests that this interface acts as a barrier to deeper penetration.

Factors contributing to the highly-localized blister formation are the high density of defects and the associated stress centers introduced near the Mo-on-Si interface by the material transition and by Mo recrystallization. For the current samples the application of post-deposition ion polishing of the Si layers, intended to control the level of roughness and interface mixing during layer deposition [69-71], will also increase the number of noble gas atoms incorporated at the top 


\section{"Ion effects in hydrogen-induced blistering of Mo/Si multilayers" \\ A. S. Kuznetsov, M. A. Gleeson and F. Bijkerk \\ Journal of Applied Physics 114(11) (2013) 113507-113512. \\ http://dx.doi.org/10.1063/1.4821844}

of the Si layers (i.e. in the vicinity of the Mo-on-Si interfaces). These impurities and the defects associated with them may serve as preferential nucleation points for blisters.

\section{“Neutral-only” blistering}

The uptake of hydrogen in the neutral-only case will be determined by the "natural" availability of dangling or stressed bond centers in the outermost Si layer. In the first instance the majority of hydrogen will go to passivating lattice dangling bonds resulting in a H-content that is effectively immobile. A small fraction of the total hydrogen in the layer will be "mobile" and will mediate transport to deeper layers. The diffusion of hydrogen in the Mo layer will be initially hindered by the presence of deep defect trap sites. However, it will increase with increasing $\mathrm{H}$-content and become orders of magnitude faster than in $\mathrm{a}$-Si. Thus the rate of vertical penetration of hydrogen through the structure will be determined primarily by the Si layers, while lateral redistribution of hydrogen will be mediated via the Mo layers.

Exposure to the neutral-only flux results in an elevated H-content primarily in the outermost Si ( 0.18 at.\%) and Mo ( 0.34 at.\%) layers (Figure 8). The content of the Si layer is comparable to the levels that were attained by deposition in a H-containing atmosphere [44] and by postdeposition ion treatment of Si layers [71]. The high content in the Mo layer can be attributed to the tendency for hydrogen to accumulate in and stabilize vacancies in the structure [31]. Segregation of multiple H-atoms to Mo mono-vacancies is energetically favorable and can lead to $\mathrm{H}_{2}$ production. Given the positive heat of solution of hydrogen in Mo and the tendency for $\mathrm{H}_{2}$ formation in high $\mathrm{H}$-content vacancies, it seems likely that segregation leading to $\mathrm{H}_{2}$ formation drives the development of "thermal neutral” blisters. As will be outlined below, the ion-induced conversion of "thermal neutral" blisters from single- to multi-component structures represents an indirect indication of $\mathrm{H}_{2}$ in these features.

\section{The effects of ions}

In light of the overview of the interactions of hydrogen with $a$-Si and metals given in the Introduction, energetic ions are anticipated to enhanced diffusion of hydrogen through the Si layers as a consequence of cleavage of existing $\mathrm{Si}-\mathrm{Si}$ and $\mathrm{Si}-\mathrm{H}$ bonds within the network. Ions will also facilitate hydrogen uptake by creating reactive trapping sites at the surface. Ionenhanced uptake is consistent with the ERD measurements, while the reduced H-content in the outermost Si layer relative to the neutral-only exposure is indicative of enhanced mobility within 


\section{"Ion effects in hydrogen-induced blistering of Mo/Si multilayers" \\ A. S. Kuznetsov, M. A. Gleeson and F. Bijkerk \\ Journal of Applied Physics 114(11) (2013) 113507-113512. \\ http://dx.doi.org/10.1063/1.4821844}

the $a$-Si network. In the interfacial regions defect creation and ion implantation can introduce new trap sites. Cleavage of bonds will aid transport within and across the interface, but may also induce relaxation of stressed bonds within the network. Ion-enhanced segregation of hydrogen to voids and vacancies (including macroscopic blisters) in the outermost Mo layer may account for the difference in H-content that is seen in the NRA data.

We now address some specific features of the current results: the appearance of the "ioninduced" blister distribution; the development of the double-/multiple-blister structures in the “thermal neutral” distribution; and the lateral damage pattern and related effects.

\section{The appearance of the "ion-induced" distribution}

At the most basic level, the appearance of the second blister distribution suggests the introduction of new nucleation sites in the ML structure. These can be the result of defect creation (vacancies or self-interstitials) or from direct implantation of ions. The ability of defects introduced in materials by ion irradiation to accumulate hydrogen is well-established. However, while induced defects and implantation will occur across the entire region probed by the penetrating ions, blister formation remains localized in the vicinity of the Mo-on-Si interfaces $[26,54]$. Thus local structural properties are the determining factor in blister manifestation, with the Mo-on-Si interface clearly providing the preferred nucleation sites.

It is noteworthy that, despite their common detachment point, the "ion-induced" and "thermal neutral” blisters distributions remain structurally distinguishable from each other. This suggests different underlying mechanisms of blister growth. The different mechanism of bubble formation that can occur in metals are near-surface plastic deformation ("blistering”), dislocation loop punching, and vacancy clustering [72]. The former two involve gaseous $\mathrm{H}_{2}$ accumulation in bubbles, while the vacancy clustering mechanism can produce voids with a relatively low molecular hydrogen content. This latter mechanism has been observed in ion-irradiated Mo [72], and is a plausible candidate for the development of the "ion-induced" blisters in the current samples. It involves the nucleation of mobile hydrogen-vacancy $(\mathrm{H}-\mathrm{V})$ complexes into higher $\mathrm{H}_{\mathrm{x}}-\mathrm{V}_{\mathrm{y}}$ complexes that acts as the blister nucleus. The initial vacancies formed by the hydrogen

ion irradiation are also stabilized by hydrogen, thereby preventing their rapid recombination with metal interstitials. 


\section{"Ion effects in hydrogen-induced blistering of Mo/Si multilayers" \\ A. S. Kuznetsov, M. A. Gleeson and F. Bijkerk \\ Journal of Applied Physics 114(11) (2013) 113507-113512. \\ http://dx.doi.org/10.1063/1.4821844}

As was noted in relation to figure 4(a), the number of "thermal neutral” blisters in the central spot increased when the exposure time was extended from 3 to 5 hours. Thus, in addition to the formation of "ion-induced" blister, ions can also induce the development of new "thermal neutral” blisters. Local strain states will be the determining factor in the type of blister that can form at a given (introduced) defect. Zhou et al [50] suggest that H-solubility in bcc metals could be enhanced by anisotropic strain almost irrespective of the sign, thereby facilitating a cascade effect of bubble formation. None-the-less, the effect was more pronounced for tensile strain values. In contrast, vacancy accumulation is energetically favorable in regions with compressive strain. Consequently, on the basis of $\mathrm{H}_{2}$ accumulation driving the "thermal neutral" blistering and vacancy clustering driving the "ion-induced" blistering, these processes would be preferentially associated with localized tensile and compressive strain centers, respectively. The existence of atomic scale regions with opposite strain values in the Mo-on-Si interface is understandable given the variations in material, composition, and crystalline state that occur during the transition. For instance, bombardment of Mo by energetic particles during deposition introduces compressive stresses, while recrystallization generates tensile stresses [73].

\section{The development of the double-/multi-component blisters}

Multi-component "thermal neutral” blisters are due to overlapping delaminations occurring at different Mo-on-Si interfaces [26]. The apparently universal conversion of these blisters from single- to double-/multi-component structures in the presence of ions is inconsistent with a random process in which the individual components develop independently. Rather, it suggests a direct connection between components. The implication is that ions are inducing transport of hydrogen across the layered structure. This can be mediated by the formation of active trapping sites at the lower surface of an initially single-component blister. Efficient transport requires an available reservoir of material to be transported. Gaseous $\mathrm{H}_{2}$ in the initial blister is a candidate for such a reservoir. Indirect evidence for the presence of $\mathrm{H}_{2}$ can be inferred from the volumearea plots shown in Figures 4 and 6. In all cases the presence of ions in the irradiating flux results in a decrease in the volume of "thermal neutral" blisters relative to their area. This is the case even while the blisters still exhibit a single-component structure (Figure 4(a); 3 hour exposure 


\section{"Ion effects in hydrogen-induced blistering of Mo/Si multilayers" \\ A. S. Kuznetsov, M. A. Gleeson and F. Bijkerk \\ Journal of Applied Physics 114(11) (2013) 113507-113512. \\ http://dx.doi.org/10.1063/1.4821844}

data). Reduction of internal gas pressure via the ion-induced transport of $\mathrm{H}_{2}$ to deeper layers would account for this effect.

\section{The lateral damage pattern}

Before discussing the origin of the lateral damage pattern, it is useful to consider the likely flux profiles of the particles that were incident on the samples during the exposures. Details of the ion profiles generated by our source are presented in the Results section. Although the neutral profiles have not been measured, several reports on the typical profiles of capillary cracker-type sources have been published [74-76]. The neutral particle profiles are typically peaked on-axis, although - depending on the capillary dimensions and operating conditions - an on-axis dip can develop in the $\mathrm{H}$-atom profile. The blistering damage in the case of the current neutral-only exposures is uniform, suggesting either a relatively flat flux profile or a high effective lateral mobility of the hydrogen that can segregate to blisters.

In any case, the neutral flux profile is clearly not the critical factor in the development of the concentric damage pattern. This pattern was only evident when the ion+neutral flux was used. It appears that the energetic ions ( $>800 \mathrm{eV}$ ) are responsible for its development, since the $<800 \mathrm{eV}$ ions have a flat lateral profile. Furthermore, comparing the diameters of the central spots seen in Figure 2 with the intensity profile widths shown in Figure 1(b) indicates that the ions in the 800$960 \mathrm{eV}$ range are the most critical for the development of the damage pattern. The relatively sharp lateral profile of these ions suggests ionization of particles in the direct flow from the capillary, implying a significant fraction of atomic ions.

The macroscopic damage pattern that develops during ion+neutral exposures is a consequence of the lateral variations in blister height and number density across the surface. The presence of energetic ions will modify the uptake and distribution of hydrogen and the local density of active defect sites. However, while the ion profiles shown in Figure 1(b) are either flat or have a simple peak shape, blister formation as a result of the ion+neutral exposures is not simply a linear function of the distance from the center of the beam spot. The individual ion profiles are insufficient to explain a pattern in which the intermediate ring is the slowest to manifest blisters. To account for such a pattern, an inter-play between different processes or between the effects of different incident species must be invoked. 


\section{"Ion effects in hydrogen-induced blistering of Mo/Si multilayers" \\ A. S. Kuznetsov, M. A. Gleeson and F. Bijkerk \\ Journal of Applied Physics 114(11) (2013) 113507-113512. \\ http://dx.doi.org/10.1063/1.4821844}

Three ion-influenced factors will affect the rate at which macroscopic blisters appear: the rate of hydrogen uptake by the ML; the rate of transport to the nucleation sites; and the local density of active nucleation sites. The presence of ions can enhance all three factors, with the effect being dependent on the local flux and the composition of the incident species. Enhanced uptake and transport rates should result in more rapid development of blisters. In contrast, the introduction of additional blister nucleation sites will have a counter-acting effect, with the growth of individual blisters being slowed by the increased number of sites consuming the available supply of hydrogen.

Enhancement of hydrogen uptake and distribution will be related to the local ion flux. The rate of introduction of new nucleation sites will also be flux dependent. However, there is a limit to the number of "ion-induced" blisters that can be formed, which is reached before the hydrogen content of the samples has stabilized [54]. Active blisters may tend to suppress the emergence of new blisters by preferential consumption of available hydrogen. This will particularly be the case if the cascade effect proposed by Zhou et al [50] is operative. This implies a shift from simultaneous site creation/activation plus blister growth during the early stages of exposure to exclusively blister growth during the later stages. Locally varying (flux and fluence dependent) H-uptake and transport rates and densities of active nucleation sites, combined with a transition from dual- to single-active processes easily provides sufficient variability to produce the observed non-linear lateral variation in the rate of blister development.

The data comparison shown in Figure 7 suggests additional complexity in the formation and development of blisters during ion+neutral exposures. The most interesting feature is the comparison between blisters formed in the periphery regions (distance $>4 \mathrm{~mm}$ ) of the samples exposed to the ion+neutral and neutral-only fluxes under otherwise identical conditions (circles and crosses, respectively). Relative to the neutral-only exposure, the areal number density is significantly increased while the average blister volume is almost halved in the case of the ion+neutral exposure. Thus a subtle ion-induced effect is also operative in the periphery. Based on Figure 1, the energetic ions incident on this region are mainly in the 960-1010 eV range and are inferred to be predominantly molecular. Hence, atomic and molecular hydrogen ions appear to have different impacts on blistering of the MLs. Molecular ions stimulate the formation of 
additional "thermal neutral” blisters but not “ion-induced” blisters (or do so less effectively than the atomic ions).

\section{Conclusions}

The influence of energetic ions on hydrogen-induced blistering of nanoscale Mo/Si MLs was investigated. A macroscopic concentric damage patterns emerges during ion+neutral exposures due to lateral variations in blister size and areal number density. These are attributed to the interplay of ion-induced variations in the rates of hydrogen uptake and distribution on the one hand, and the number density of active blister nucleation points on the other. Based on comparison of the effects induced by exposure to incident neutral-only and ion+neutral hydrogen fluxes, outline mechanisms for the observed development of two distinct blister distributions are proposed. The first involves hydrogen accumulation in voids and vacancies in Mo leading to the growth of $\mathrm{H}_{2}$ filled bubbles. This can occur in the absence of ions and is driven by energetically favorable segregation of hydrogen to Mo vacancies. The second mechanism is a $\mathrm{H}-\mathrm{V}$ clustering process that require energetic ions in order to produce the initiating stabilized vacancy complexes. Both processes lead to the formation of blisters that are localized at the Mo-on-Si interfaces. This localization is dictated by the strain centers and defects that develop during the formation of these interfaces.

In addition to stimulating the $\mathrm{H}-\mathrm{V}$ clustering mechanism, energetic ions induce the formation of additional $\mathrm{H}_{2}$-filled blisters. They also promote the uptake and distribution of hydrogen within the ML. These effects are particularly relevant to transport across the Si layers. The formation of complex blister structures with overlapping delaminations at different interfaces is attributed to ion-induced transport of hydrogen trapped in gas-filled bubbles across the outermost Mo-on-Si interface.

\section{Acknowledgements}

The authors would like to thank F. Boekhout and Dr. R.W.E. van de Kruijs for sample deposition and Dr. G. de Temmerman for advice and useful discussions. This work is a part of XMO ('eXtreme Multilayer Optics) funded by FOM (Fundamental Onderzoek der Materie) and the EXEPT (Extreme UV lithography Entry Point Technology development) programme of 


\section{"Ion effects in hydrogen-induced blistering of Mo/Si multilayers" A. S. Kuznetsov, M. A. Gleeson and F. Bijkerk \\ Journal of Applied Physics 114(11) (2013) 113507-113512. http://dx.doi.org/10.1063/1.4821844}

CATRENE, supported by the AgentschapNL. NRA measurements were carried out at the Institute of Ion Beam Physics and Materials Research from HZDR (Helmholtz-Zentrum Dresden-Rossendorf) in Dresden. RBS and ERD measurements were performed by AccTec BV, Eindhoven. Dr. W.M. Arnold Bik is gratefully acknowledged for his assistance in obtaining the NRA, RBS, and ERD analyses.

\section{References.}

[1] C. Braley, F. Mazen, A. Tauzin, F. Rieutord, C. Deguet, E. Ntsoenzok, Nucl. Instru. Meth. Phys. Res. B 277 (2012) 93.

[2] A.Y. Usenko, A.G. Ulyashin, Jpn. J. Appl. Phys. Part 1 - Regul. Pap. Short Notes Rev. Pap. 41/8 (2002) 5021.

[3] W. Düngen, R. Job, T. Mueller, Y. Ma, W.R. Fahrner, L.O. Keller, J.T. Horstmann, H. Fiedler, J. Appl. Phys. 100/12 (2006) 124906

[4] M. Balden, S. Lindig, A. Manhard, J.H. You, J. Nucl. Mater. 414/1 (2011) 69.

[5] A.F. Bardamid, K.Y. Vukolov, V.G. Konovalov, D.I. Naidenkova, A.V. Rogov, I.V. Ryzhkov, A.N. Shapoval, A.F. Shtan, S.I. Solodovchenko, V.S. Voitsenya, K.I. Yakimov, Plasma Devices Oper. 14/2 (2006) 159.

[6] G. De Temmerman, R.P. Doerner, J. Nucl. Mater. 389/3 (2009) 479.

[7] S. Lindig, M. Balden, V.K. Alimov, T. Yamanishi, W.M. Shu, J. Roth, Phys. Scr. T138 (2009) 014040.

[8] W.M. Shu, Appl. Phys. Lett. 92/21 (2008) 211904.

[9] B. Audoly, Phys. Rev. Lett. 83/20 (1999) 4124.

[10] C. Coupeau, J. Grilhe, E. Dion, L.D. de Morais, J. Colin, Thin Solid Films 518/18 (2010) 5233.

[11] P. Goudeau, M. George, C. Coupeau, Applied Physics Letters 84/6 (2004) 894.

[12] Z.F. Di, Y.Q. Wang, M. Nastasi, F. Rossi, L. Shao, P.E. Thompson, Appl. Phys. Lett. 93/25 (2008) 254104.

[13] X.B. Ma, X.F. Du, W.L. Liu, C. Chen, Z.T. Song, C.L. Lin, J. Vac. Sci. Technol. B 27/3 (2009) 1063. 


\section{"Ion effects in hydrogen-induced blistering of Mo/Si multilayers" A. S. Kuznetsov, M. A. Gleeson and F. Bijkerk \\ Journal of Applied Physics 114(11) (2013) 113507-113512. \\ http://dx.doi.org/10.1063/1.4821844}

[14] L. Shao, Y. Lin, J.K. Lee, Q.X. Jia, Y.Q. Wang, M. Nastasi, P.E. Thompson, N.D. Theodore, P.K. Chu, T.L. Alford, J.W. Mayer, P. Chen, S.S. Lau, Appl. Phys. Lett. 87/9 (2005) 091902.

[15] L. Shao, Y. Lin, J.G. Swadener, J.K. Lee, Q.X. Jia, Y.Q. Wang, M. Nastasi, P.E. Thompson, N.D. Theodore, T.L. Alford, J.W. Mayer, P. Chen, S.S. Lau, Appl. Phys. Lett. 87/25 (2005) 251907.

[16] F. Okba, N. Cherkashin, Z. Di, M. Nastasi, F. Rossi, A. Merabet, A. Claverie, Appl. Phys. Lett. 97/3 (2010) 031917.

[17] M.J. Demkowicz, D. Bhattacharyya, I. Usov, Y.Q. Wang, M. Nastasi, A. Misra, Appl. Phys. Lett. 97/16 (2010) 161903.

[18] K. Hattar, M.J. Demkowicz, A. Misra, I.M. Robertson, R.G. Hoagland, Scripta Materialia 58/7 (2008) 541.

[19] T. Hochbauer, A. Misra, K. Hattar, R.G. Hoagland, J. Appl. Phys. 98/12 (2005) 123516.

[20] N. Li, M.S. Martin, O. Anderoglu, A. Misra, L. Shao, H. Wang, X. Zhang, J. Appl. Phys. 105/12 (2009) 123522.

[21] C. Frigeri, M. Serenyi, N.Q. Khanh, A. Csik, F. Riesz, Z. Erdelyi, L. Nasi, D.L. Beke, H.G. Boyen, Nanoscale Res. Lett. 6 (2011) 189.

[22] C. Frigeri, L. Nasi, M. Serenyi, A. Csik, Z. Erdelyi, D.L. Beke, Superlattices and Microstructures 45/4-5 (2009) 475.

[23] W.P. Kalisvaart, A. Kubis, M. Danaie, B.S. Amirkhiz, D. Mitlin, Acta Materialia 59/5 (2011) 2083.

[24] M.G. Pelizzo, A.J. Corso, P. Zuppella, D.L. Windt, G. Mattei, P. Nicolosi, Optics Express 19/16 (2011) 14838.

[25] K. Munbodh, F.A. Perez, D. Lederman, J. Appl. Phys. 111/12 (2012) 123919.

[26] A.S. Kuznetsov, M.A. Gleeson, F. Bijkerk, J. Phys.: Condens. Matter 24/5 (2012) 052203.

[27] C.G. Van de Walle, J. Neugebauer, Annual Review of Materials Research, vol. 36, Annual Reviews, Palo Alto, 2006, p. 179.

[28] S. Chakraborty, D.A. Drabold, Phys. Rev. B 79/11 (2009) 115214.

[29] T. Hoshihira, T. Otsuka, T. Tanabe, J. Nucl. Mater. 390-91 (2009) 1029. 


\section{"Ion effects in hydrogen-induced blistering of Mo/Si multilayers" A. S. Kuznetsov, M. A. Gleeson and F. Bijkerk \\ Journal of Applied Physics 114(11) (2013) 113507-113512. http://dx.doi.org/10.1063/1.4821844}

[30] R. Griessen, Physical Review B 38/6 (1988) 3690.

[31] K. Ohsawa, K. Eguchi, H. Watanabe, M. Yamaguchi, M. Yagi, Phys. Rev. B 85/9 (2012) 094102.

[32] B.R. Tuttle, Mod. Phys. Lett. B 19/15 (2005) 683.

[33] N.H. Nickel, K. Brendel, Appl. Phys. Lett. 82/18 (2003) 3029.

[34] C.G. van de Walle, Physical Review B 49/7 (1994) 4579.

[35] P.A. Fedders, Physical Review B 61/23 (2000) 15797.

[36] Y.S. Su, S.T. Pantelides, Phys. Rev. Lett. 88/16 (2002) 165503.

[37] T.A. Abtew, F. Inam, D.A. Drabold, Epl 79/3 (2007) 36001.

[38] T. Sadoh, R. Matsuura, M. Miyao, M. Ninomiya, M. Nakamae, T. Enokida, Appl. Phys. Lett. 86/21 (2005) 211901.

[39] Z.F. Di, Y.Q. Wang, M. Nastasi, L. Shao, J.K. Lee, N.D. Theodore, Appl. Phys. Lett. 93/10 (2008) 104103

[40] R.A. Andrievski, Phys. Usp. 50/7 (2007) 691.

[41] A. Pundt, Advanced Engineering Materials 6/1-2 (2004) 11.

[42] A.V. Gapontsev, V.V. Kondrat'ev, Phys. Usp. 46/10 (2003) 1077.

[43] R. Kirchheim, T. Mütschele, W. Kieninger, H. Gleiter, R. Birringer, T.D. Koblé, Materials Science and Engineering 99/1-2 (1988) 457.

[44] V. Rigato, A. Patelli, G. Maggioni, G. Salmaso, V. Mattarello, M.G. Pelizzo, P. Nicolosi, L. Depero, E. Bontempi, P. Mazzoldi, Surf. Coat. Technol. 174 (2003) 40.

[45] S.M. Myers, F. Besenbacher, J. Appl. Phys. 60/10 (1986) 3499.

[46] J. Bottiger, S.T. Picraux, N. Rud, T. Laursen, J. Appl. Phys. 48/3 (1977) 920.

[47] S. Nagata, B. Tsuchiya, N. Ohtsu, T. Sugawara, T. Shikama, K. Tokunaga, M. Takenaka, E. Kuramoto, J. Nucl. Mater. 313 (2003) 279.

[48] S. Nagata, K. Tokunaga, B. Tsuchiya, N. Ohtsu, T. Sugawara, T. Shikama, J. Alloy. Compd. 356 (2003) 326.

[49] Y. Fukai, J. Alloy. Compd. 356 (2003) 263.

[50] H.B. Zhou, S. Jin, Y. Zhang, G.H. Lu, F. Liu, Phys. Rev. Lett. 109/13 (2012) 135502.

[51] B. Hjörvarsson, G. Andersson, E. Karlsson, J. Alloy. Compd. 253 (1997) 51.

[52] A. Pundt, R. Kirchheim, Annual Review of Materials Research, vol. 36, 2006, p. 555. 


\section{"Ion effects in hydrogen-induced blistering of Mo/Si multilayers" A. S. Kuznetsov, M. A. Gleeson and F. Bijkerk \\ Journal of Applied Physics 114(11) (2013) 113507-113512. \\ http://dx.doi.org/10.1063/1.4821844}

[53] D.L. Windt, W.L. Brown, C.A. Volkert, W.K. Waskiewicz, J. Appl. Phys. 78/4 (1995) 2423.

[54] A.S. Kuznetsov, M.A. Gleeson, F. Bijkerk, Thin Solid Films, 545 (2013) 571-579.

[55] A.S. Kuznetsov, M.A. Gleeson, R.W.E.v.d. Kruijs, F. Bijkerk, Proc. SPIE 8077/1 (2011) 807713.

[56] G.K. Celler, S. Cristoloveanu, J. Appl. Phys. 93/9 (2003) 4955.

[57] M. Bruel, Nucl. Instrum. Methods Phys. Res. Sect. B-Beam Interact. Mater. Atoms 108/3 (1996) 313.

[58] R.C. Wieggers, W.J. Goedheer, M.R. Akdim, F. Bijkerk, P.A. Zegeling, J. Appl. Phys. 103/1 (2008) 013308.

[59] I. Nedelcu, R. de Kruijs, A.E. Yakshin, F. Tichelaar, E. Zoethout, E. Louis, H. Enkisch, S. Muellender, F. Bijkerk, Thin Solid Films 515/2 (2006) 434.

[60] V.I.T.A. de Rooij-Lohmann, I.V. Kozhevnikov, L. Peverini, E. Ziegler, R. Cuerno, F. Bijkerk, A.E. Yakshin, Appl. Surf. Sci. 256 (2010) 5011.

[61] Oxford Applied Research Thermal Cracker TC 50, http://www.oaresearch.co.uk/oaresearch/tc50/Default.aspx

[62] I. Nedelcu, R.W.E. van de Kruijs, A.E. Yakshin, F. Bijkerk, Phys. Rev. B 76/24 (2007) 245404.

[63] S. Bruijn, R.W.E. van de Kruijs, A.E. Yakshin, F. Bijkerk, Applied Surface Science 257/7 (2011) 2707.

[64] R.W.E. van de Kruijs, E. Zoethout, A.E. Yakshin, I. Nedelcu, E. Louis, H. Enkisch, G. Sipos, S. Mullender, F. Bijkerk, Thin Solid Films 515/2 (2006) 430.

[65] J. Bosgra, J. Verhoeven, R.W.E. van de Kruijs, A.E. Yakshin, F. Bijkerk, Thin Solid Films 522 (2012) 228.

[66] S. Bajt, D.G. Stearns, P.A. Kearney, J. Appl. Phys. 90/2 (2001) 1017.

[67] E.N. Zubarev, V.V. Kondratenko, Y.P. Pershyn, V.A. Sevryukova, Thin Solid Films 520/1 (2011) 314.

[68] E. Zoethout, G. Sipos, R. van de Kruijs, A. Yakshin, E. Louis, S. Muellender, F. Bijkerk, Proc. SPIE 5037 (2003) 872.

[69] X.W. Zhou, H.N.G. Wadley, J. Appl. Phys. 87/12 (2000) 8487. 


\section{"Ion effects in hydrogen-induced blistering of Mo/Si multilayers" A. S. Kuznetsov, M. A. Gleeson and F. Bijkerk \\ Journal of Applied Physics 114(11) (2013) 113507-113512. \\ http://dx.doi.org/10.1063/1.4821844}

[70] X.W. Zhou, H.N.G. Wadley, J. Appl. Phys. 88/10 (2000) 5737.

[71] R. Schlatmann, A. Keppel, Y. Xue, J. Verhoeven, C.H.M. Maree, F. Habraken, J. Appl. Phys. 80/4 (1996) 2121.

[72] J.B. Condon, T. Schober, J. Nucl. Mater. 207 (1993) 1.

[73] V.I. Pinegyn, E.N. Zubarev, V.V. Kondratenko, V.A. Sevryukova, S.A. Yulin, T. Feigl, N. Kaiser, Thin Solid Films 516/10 (2008) 2973.

[74] T. Schwarz-Selinger, A. von Keudell, W. Jacob, J. Vac. Sci. Technol. A-Vac. Surf. Films 18/3 (2000) 995.

[75] K.G. Tschersich, J. Appl. Phys. 87/5 (2000) 2565.

[76] K.G. Tschersich, V. von Bonin, J. Appl. Phys. 84/8 (1998) 4065. 

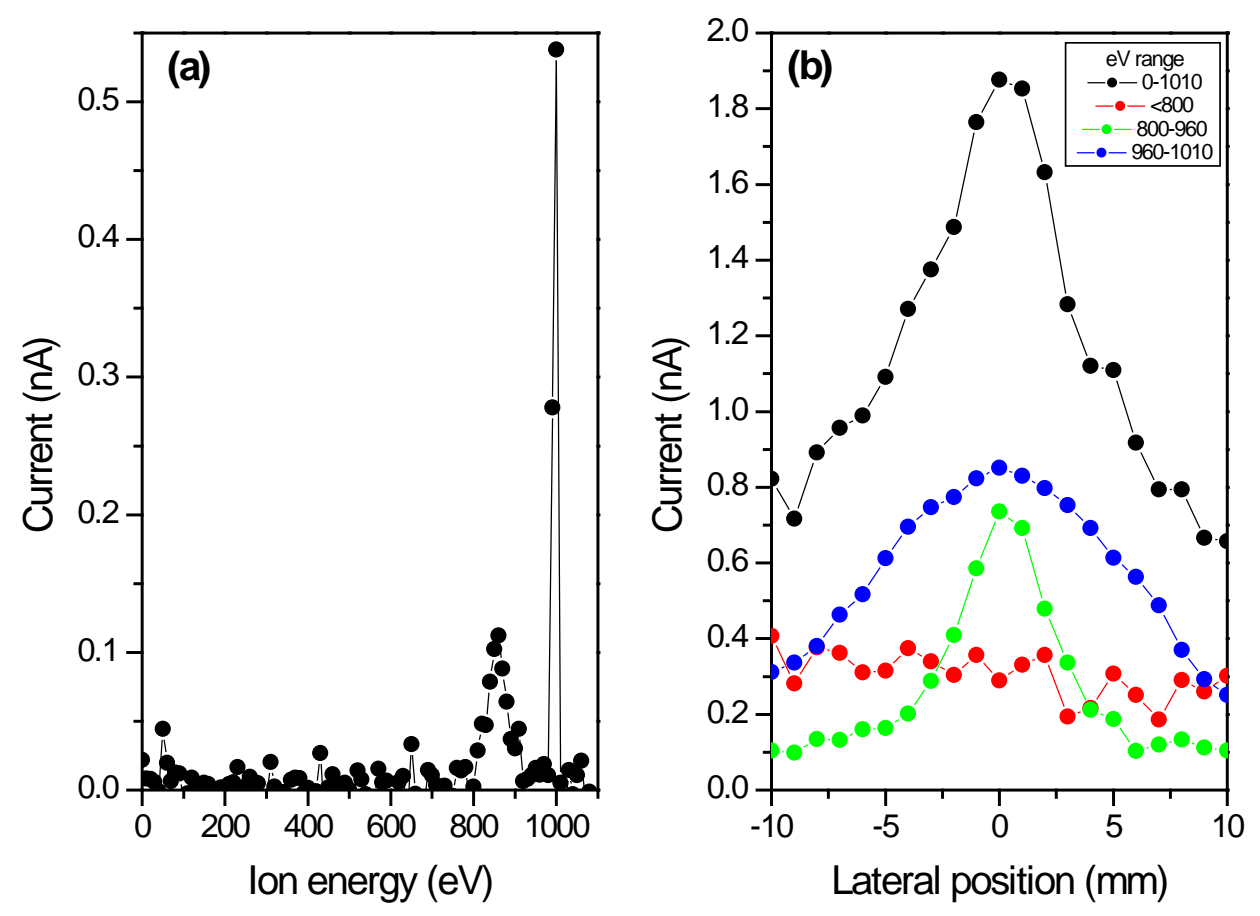

Figure 1: Ion current profiles from the capillary cracker for a $\mathrm{H}_{2}$ gas flow of 1 sccm. (a) Current as a function of energy measured along the central axis position of the capillary cracker beam; (b) Current as a function of lateral distance from the central axis position for selected ion energy ranges. 


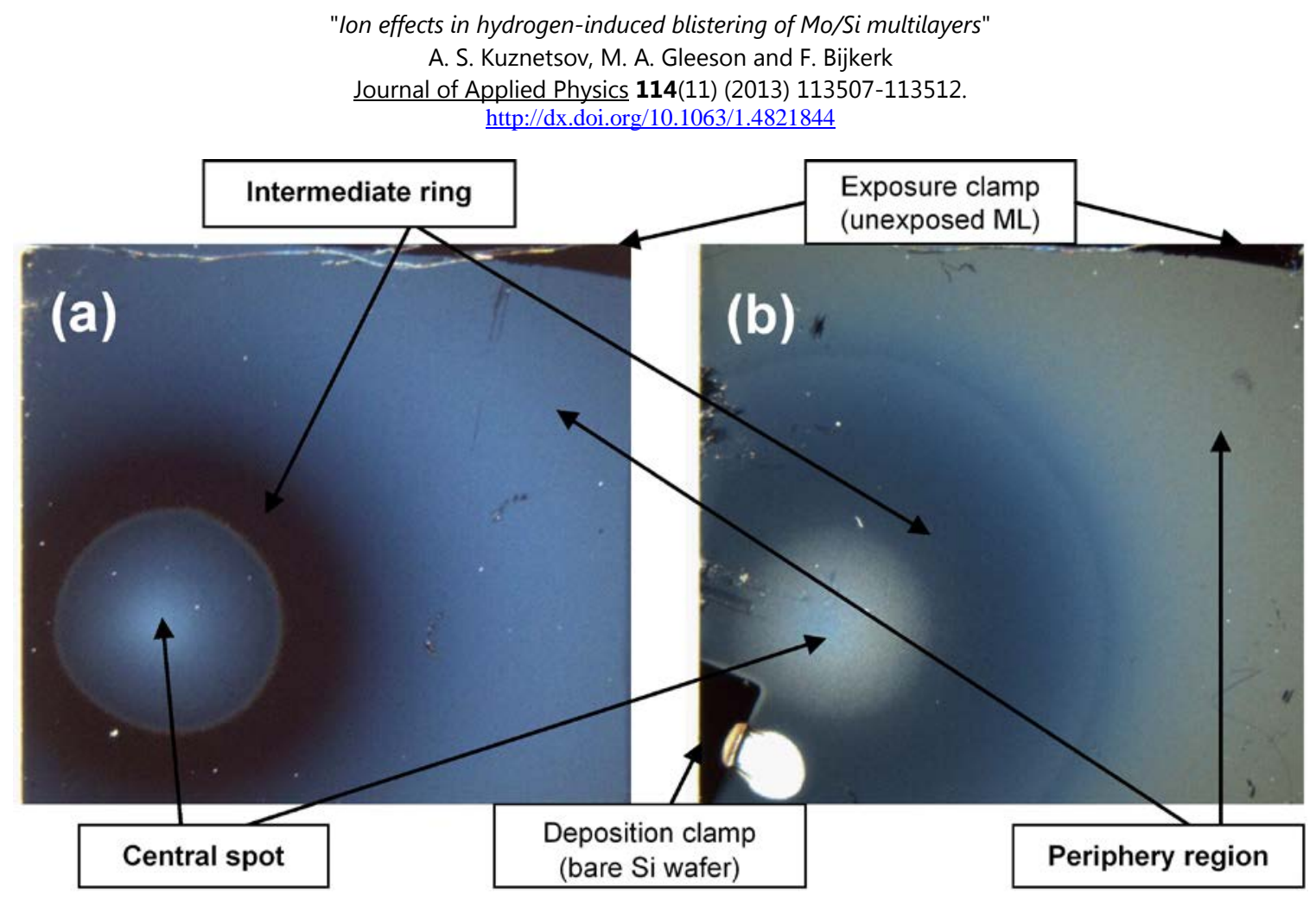

Figure 2: Optical images of $\sim 12 \times 12 \mathrm{~mm}^{2}$ ML samples after exposure for (a) 3 hours and (b) 5 hours to the unmodified hydrogen flux from the thermal capillary cracker at $1 \mathrm{sccm} \mathrm{H}_{2}$ flow ( $\left.\mathrm{T}_{\text {exposure }} \sim 298-358 \mathrm{~K}\right)$. 

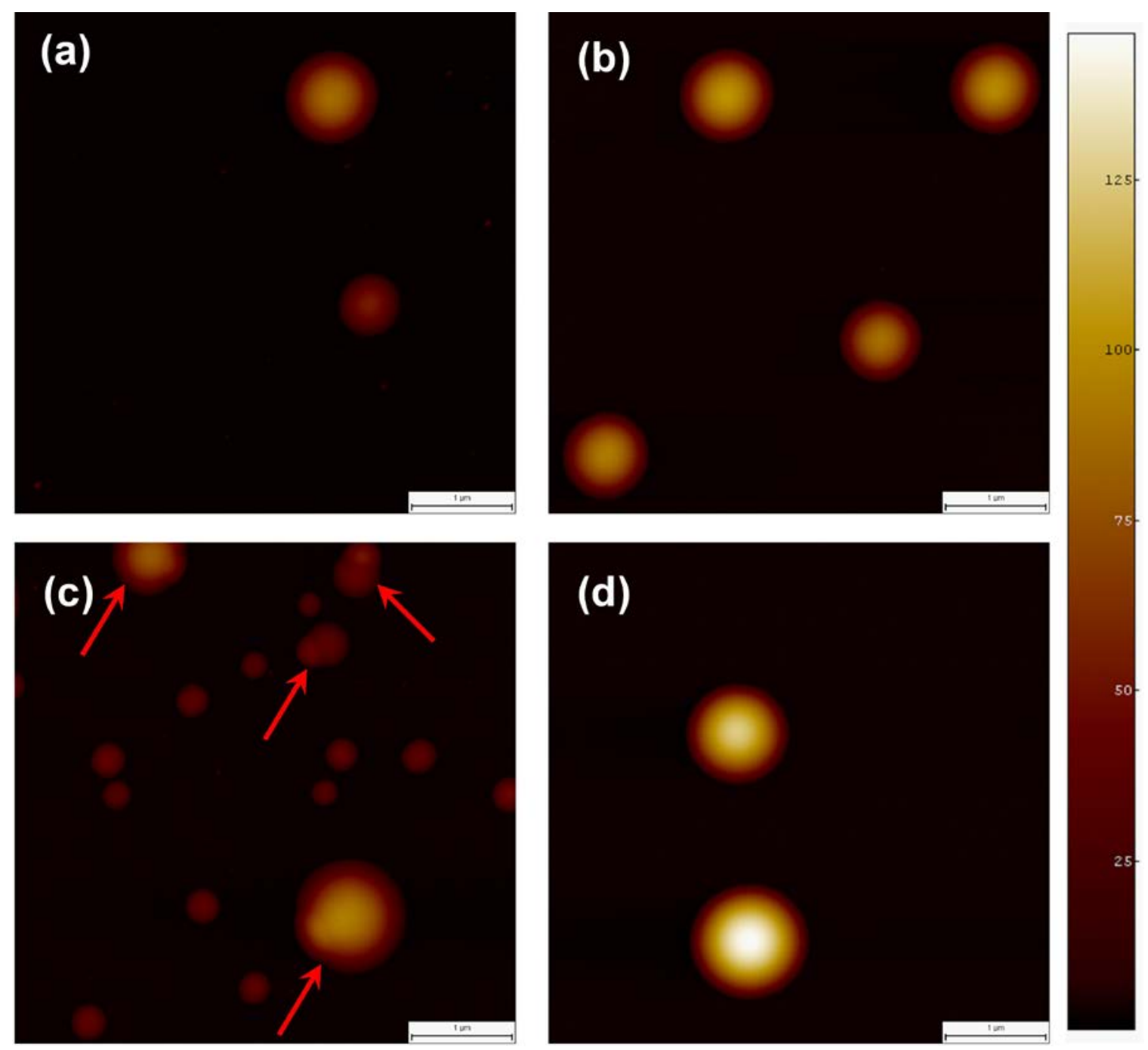

Figure 3: $5 \mathrm{x} 5 \mu^{2}$ AFM images collected from (a) the central spot and (b) the periphery region of the 3-hour exposed sample shown in figure 2(a); and from (c) the central spot and (d) the periphery region of the 5-hour exposed sample shown in figure 2(b). The arrows on panel (c) indicate “double-blister” structures. The Z-scale is common to all images. 

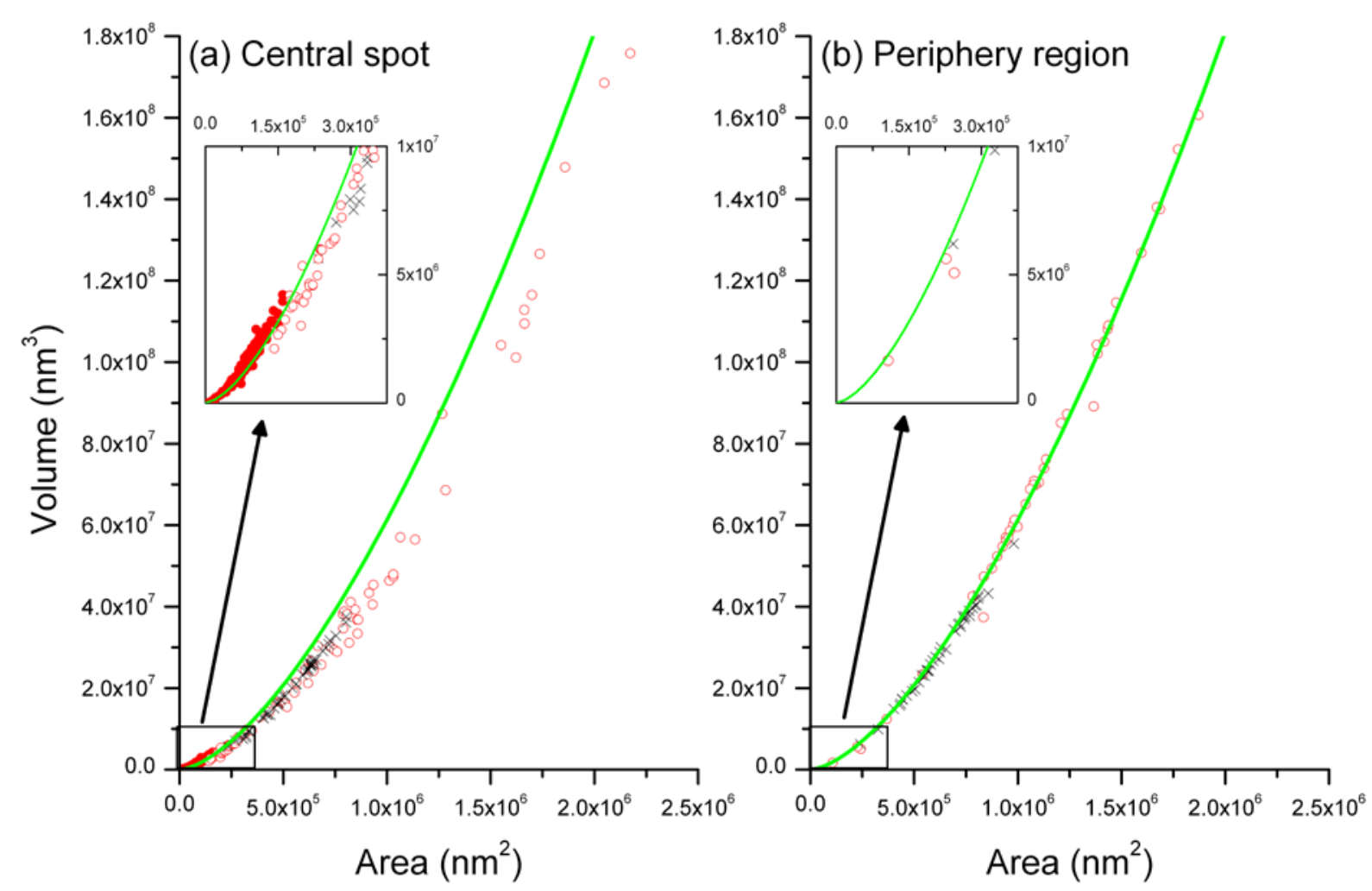

Figure 4: Plots of individual blister volume versus surface area covered derived from $25 \times 25$ $\mu \mathrm{m}^{2}$ AFM scans: (a) central spot and (b) periphery region. The data from the samples exposed for 3 and 5 hours are indicated with crosses and circles, respectively. In the case of the 5-hour exposure data, filled circles represent "ion-induced" blisters and open circles represent "thermal neutral” blisters. The solid line was obtained by fitting the 5-hour periphery region data with the function: $\mathrm{y}=\mathrm{a} \times\left(\mathrm{x}^{\mathrm{b}}\right)$. The insets on the two panels are blow-ups of the dimension range containing the "ion-induced" blisters. 

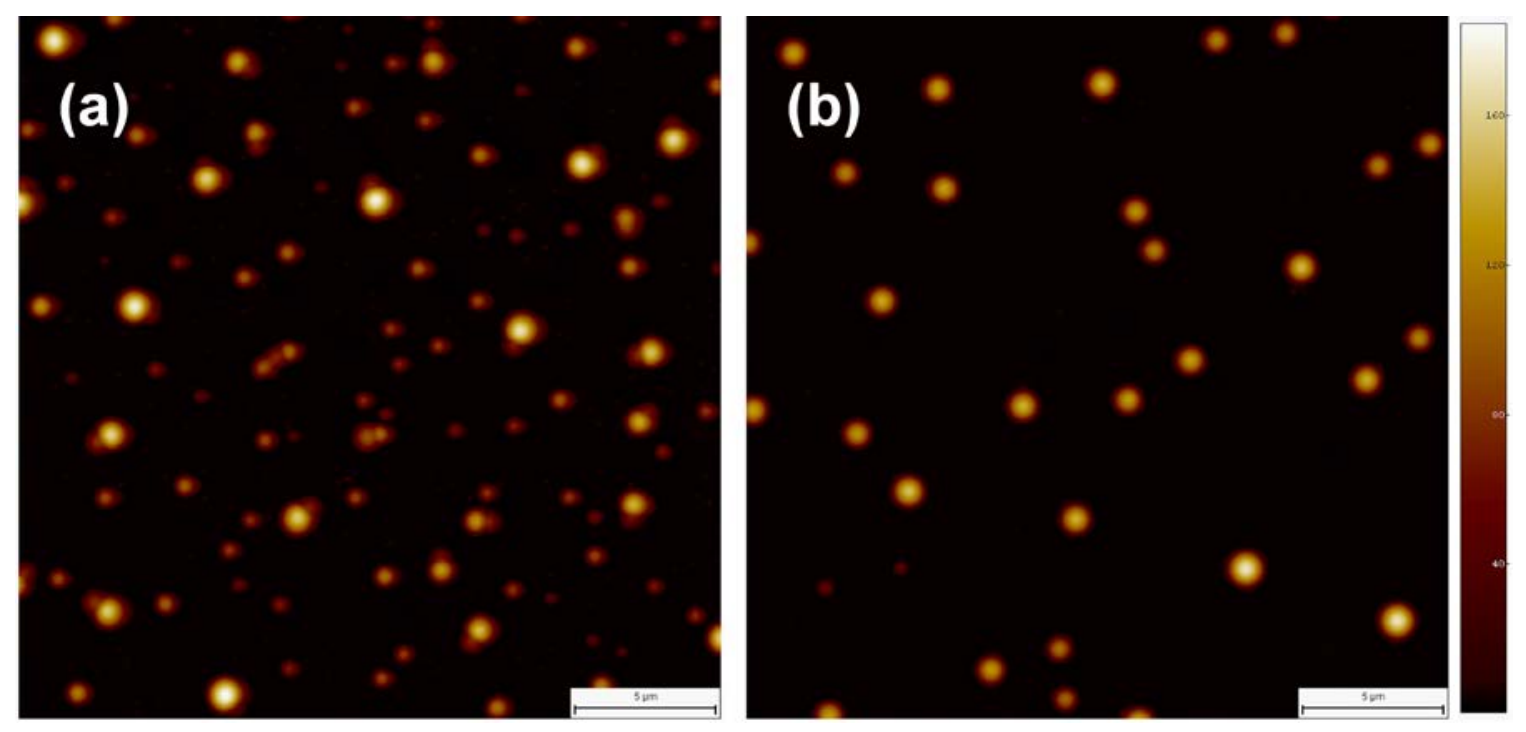

Figure 5: 25x25 $\mu \mathrm{m}^{2}$ AFM images collected from the central spot position of samples after 5 hour exposures to (a) the "ion+neutral" and (b) the "neutral-only" flux from the thermal capillary cracker at $1 \mathrm{sccm} \mathrm{H}_{2}$ flow ( $\mathrm{T}_{\text {exposure }} \sim 373 \mathrm{~K}$ ). The Z-scale is common to both images. 


\section{"Ion effects in hydrogen-induced blistering of Mo/Si multilayers" \\ A. S. Kuznetsov, M. A. Gleeson and F. Bijkerk \\ Journal of Applied Physics 114(11) (2013) 113507-113512. \\ http://dx.doi.org/10.1063/1.4821844}
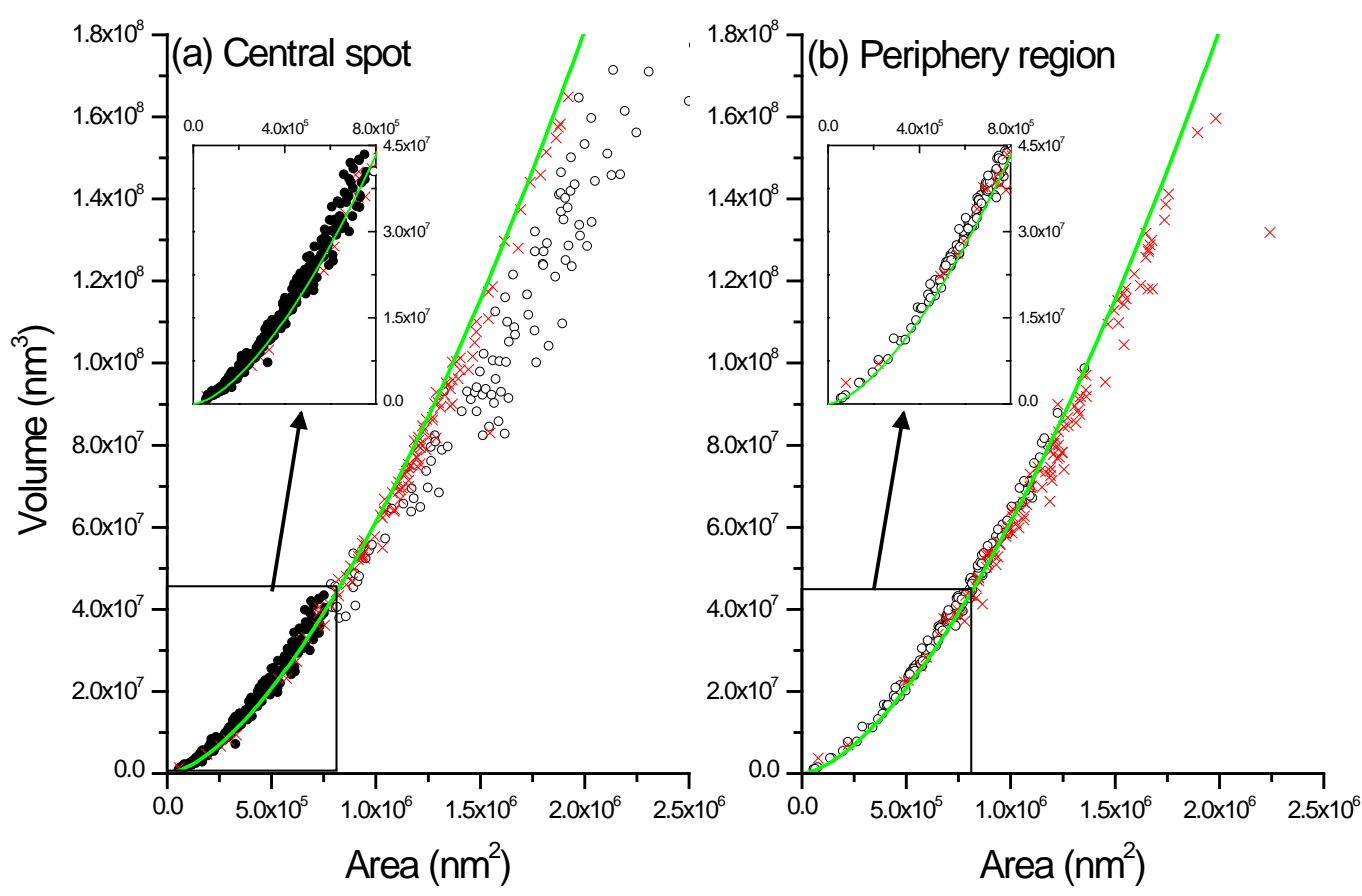

Figure 6: Plots of individual blister volume versus area covered derived from $50 \times 50 \mu \mathrm{m}^{2}$ AFM scans of (a) the central spot and (b) the periphery region of "ion+neutral” (circles) and "neutralonly” (crosses) exposed samples. In the case of the ion+neutral data, "ion-induced” blisters are represented by filled circles and “thermal neutral” blisters by open circles. The solid lines reproduce the curve that was derived from figure 4(b). The insets on the two panels are blow-ups of the dimension range containing the "ion-induced" blisters. 

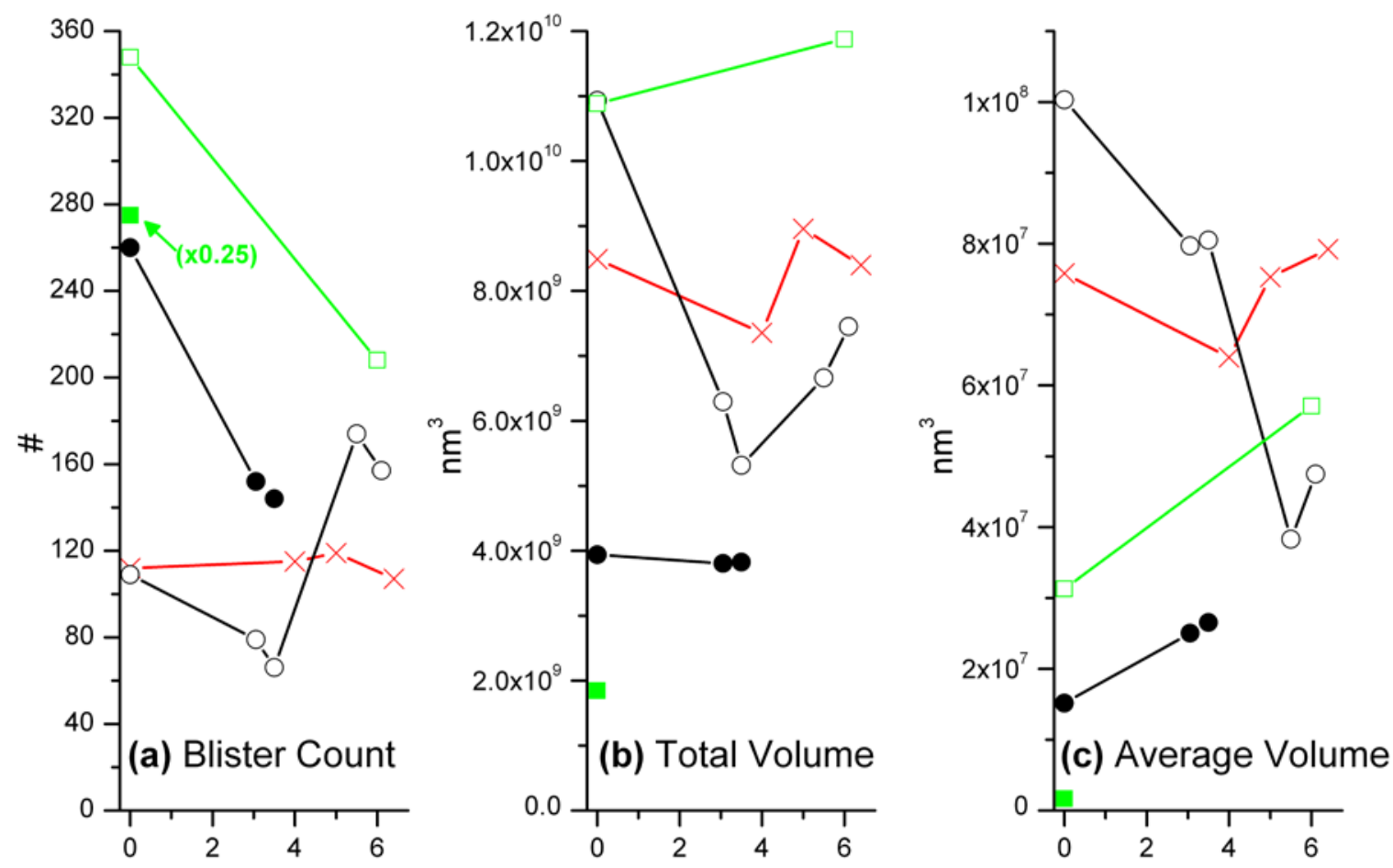

Distance $(\mathrm{mm})$

Figure 7: Plots of (a) blister count, (b) total blister volume, and (c) average blister volume derived from AFM scans of the three 5 hour exposed samples. Data from the "ion+neutral” and “neutral-only” exposed samples (Figures 5-6) are indicate by circles and crosses, respectively and are based on analysis of 50x50 $\mu \mathrm{m}^{2}$ scans. Squares represent data from the "original” 5 hour ion+neutral exposure (Figures 2-4) and are based on 25x25 $\mu \mathrm{m}^{2}$ scans. These blister count and total volume values have been correct for the difference in scan size. In the case of the two ion+neutral exposures, “ion-induced” and "thermal neutral” distributions are represented by filled and open symbols, respectively. The corrected "ion-induced” blister count from the original ion+neutral exposure has been reduced by a factor of four. The $\mathrm{x}$-axis distances are relative to the approximate centre of the “central spot” position. 


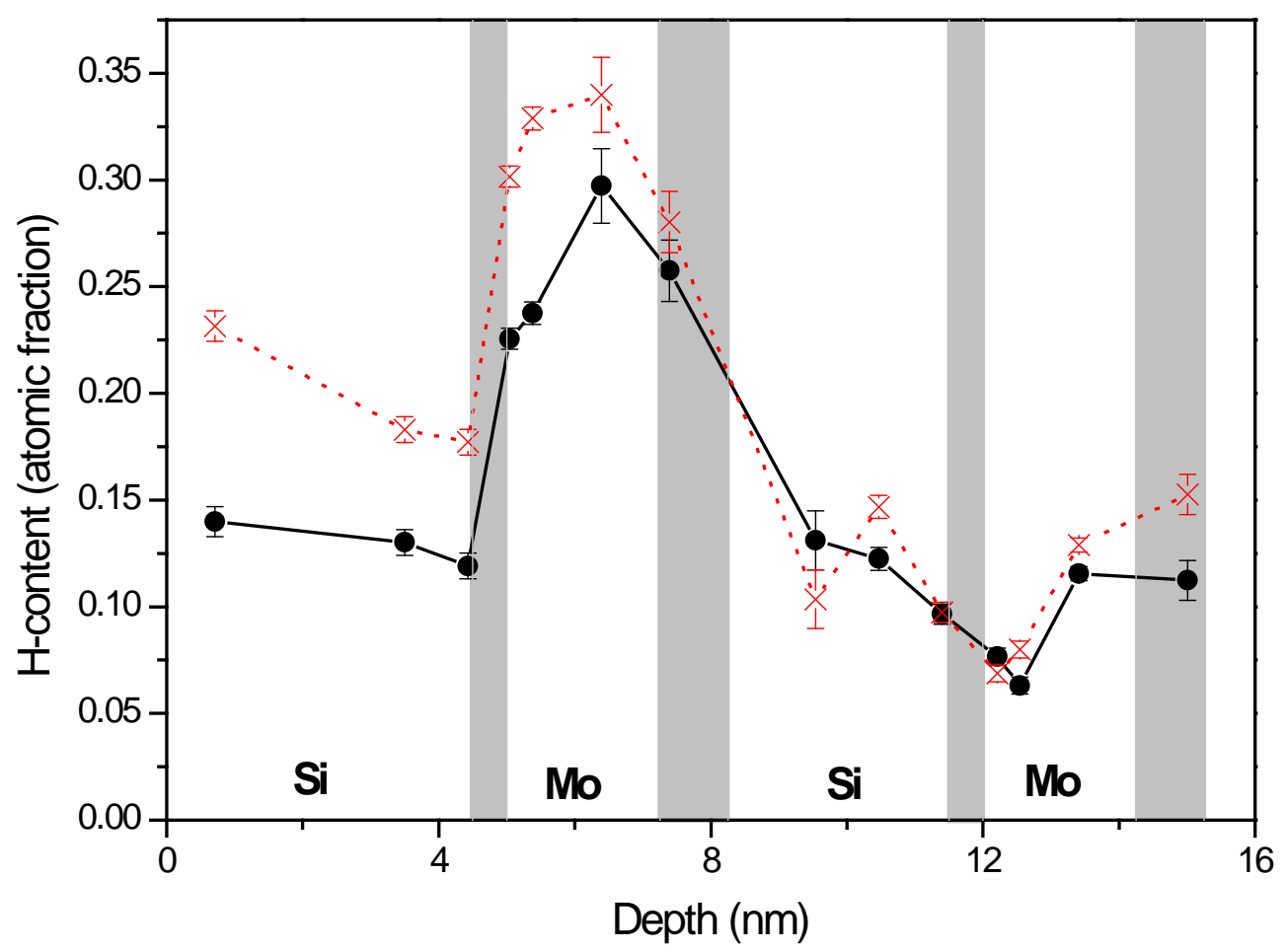

Figure 8: Resonant NRA depth profiles of the H-content of samples after exposure to the "ion+neutral" (circles) and "neutral-only" (crosses) fluxes from the thermal capillary cracker. The approximate layer structure is indicated. The shaded regions represent interfacial zones. 